\title{
Transfusiones de glóbulos rojos en recién nacidos de muy bajo peso de nacimiento
}

\author{
ISABEL FOLATRE B. ${ }^{1,2}$, CARLA KUSCHEL B. ${ }^{3}$, FRANCISCO MARÍN H. ${ }^{4}$ \\ 1. Instituto de Pediatría, Facultad de Medicina, Universidad Austral de Chile, Valdivia. \\ 2. Subdepartamento de Pediatría, Hospital Base Valdivia. \\ 3. Facultad de Medicina, Universidad Austral de Chile, Valdivia. \\ 4. Bioestadístico, Instituto de Salud Pública, Facultad de Medicina, Universidad Austral de Chile, Valdivia.
}

\begin{abstract}
\section{Red blood cell transfusions in very low birth weight newborns}

Introduction: It has been reported that $80 \%$ of very low birth weight infants (VLBWI) are receiving packed red blood cell transfusions (PRBCtr), and in $90 \%$ of cases, the indication is the replacement of the blood collected. The existence of guidelines for transfusion practices has had a great impact on the decline in the number of transfusions. The aim of this paper is to describe the characteristics of VLBW infants who are packed red blood cell transfusion receptors. Patients and Methods: This is a descriptive cross-sectional study, which included the medical records of all VLBW newborns older than 72 hours, released from the Neonatology department of the Hospital Valdivia Base, between 2005 and 2006. Birth weight, gestational age, pulmonary surfactant, hyaline membrane, mechanical ventilation, bronchopulmonary dysplasia, sepsis and packed red blood cell transfusions were evaluated. Guidelines for packed red cell transfusions were used at the Hospital. Results: 93 newborns were evaluated and 62 of them were transfused (66.7\%); they received $2.1 \pm 0.9$ PRBC transfusion and the exposure to different donors was $2.1 \pm 0.9$. The VLBW infants susceptible to be PRBCtr receptor were those younger than 30 weeks' gestational age, weighing less than $1,250 \mathrm{~g}$ and with respiratory failure, mechanical ventilation and sepsis. Conclusion: The observed percentages of transfused infants with very low birth weight, median PRBCtr and exposure to different donors can be attributed to the existence of guidelines for neonatal transfusion practices and a team of highly experienced neonatologists.
\end{abstract}

(Key words: Very low birth weight newborn, transfusion, anemia, red blood cell).

Rev Chil Pediatr 2014; 85 (3): 298-303

\section{RESUMEN}

Introducción: Se ha reportado que el $80 \%$ de los recién nacidos de muy bajo peso de nacimiento (RNMBPN) es receptor de transfusiones de glóbulos rojos desplasmatizados (trGRD) y en el 90\% de ellos, la indicación de transfusión es la reposición de sangre extraída. La acción en salud que mayor impacto ha tenido sobre el descenso del número de transfusiones, es la existencia de guías para la práctica transfusional. El objetivo de este trabajo es describir las características de los RNMBPN receptores de trGRD. Pacientes y Método: Estudio transversal descriptivo, que incluyó las fichas clínicas de todos los RNMBPN, mayores de $72 \mathrm{~h}$ de vida, egre-

Recibido el 01 de diciembre de 2012, devuelto para corregir el 20 de marzo de 2013, segunda versión 06 de julio de 2013 , tercera versión 15 de enero de 2014, aceptado para publicación el 07 de febrero de 2014.

Este trabajo cumple con los requisitos sobre consentimiento /asentimiento informado, comité de ética, financiamiento, estudios animales y sobre la ausencia de conflictos de intereses según corresponda.

Correspondencia a:

Isabel Folatre B.

E-mail: ifolatre@uach.cl 
sados de Neonatología, Hospital Base Valdivia, entre el año 2005 y 2006. Se evaluaron el peso de nacimiento, edad gestacional, surfactante pulmonar, membrana hialina, ventilación mecánica, displasia broncopulmonar, sepsis y transfusiones de GRD. Se utilizó la Guía para la Práctica Transfusional de GRD en neonatos del Centro Base. Resultados: Fueron evaluados 93 neonatos, siendo transfundidos 62 de 93 neonatos $(66,7 \%)$ recibiendo $2,1 \pm 0,9$ trGRD, la exposición a donantes diferentes fue de 2,1 $\pm 0,9$. Los RNMBPN susceptible de ser receptor de trGRD fueron de edad gestacional $<30 \mathrm{sem}$, peso inferior a $1.250 \mathrm{~g}$, con falla respiratoria, ventilación mecánica y sepsis. Conclusión: Los porcentajes observados de RNMBPN transfundidos, la media de trGRD y de exposición a diferentes donantes, podrían ser atribuidos a la existencia de guías para la práctica transfusional neonatal y a un equipo de neonatólogos altamente sensibilizado.

(Palabras clave: Recién nacido de muy bajo peso de nacimiento, transfusión, anemia, globulos rojos).

Rev Chil Pediatr 2014; 85 (3): 298-303

\section{Introducción}

Los recién nacidos de muy bajo peso de nacimiento (RNMBPN) presentan anemia por una coexistencia de factores, entre ellos, vida media acortada de los eritrocitos, con un descenso observado en la concentración de hemoglobina de $1 \mathrm{~g} \%$ desde la segunda a la octava semanas de vida, y producción inadecuada de eritropoyetina para una concentración dada de hemoglobina, resultante en una eritropoyesis cuantitativamente insuficiente ${ }^{1-2}$.

Las unidades de cuidado intensivo neonatal (UCIN) han permitido la sobrevida de neonatos cada vez más pequeños, con exposición a un número importante de extracciones sanguíneas durante las primeras semanas de vida. Strauss describió que el $80 \%$ de este grupo de pacientes fue receptor de transfusiones de glóbulos rojos desplasmatizados (trGRD) y en el $90 \%$ de ellos, la indicación de transfusión fue reposición de sangre extraída ${ }^{1-2}$.

Siendo la volemia estimada de los RNMBPN de alrededor de $100 \mathrm{ml} / \mathrm{kg}$ de peso, el volumen de sangre extraído tiene un impacto mayor sobre el volumen circulante; este grupo etáreo compensa la hipovolemia en forma diferente a los adultos y al disminuir la volemia en $10 \%$, se reduce el bombeo ventricular izquierdo, sin aumentar la frecuencia cardíaca, logrando preservar la presión arterial sistémica mediante aumento de la resistencia vascular periférica, resultando en deterioro de la perfusión y oxigenación tisulares, más acidosis metabólica ${ }^{3}$.
Lagos, Hering y cols., describieron en nuestro medio que el $89,4 \%$ de los RNMBPN evaluados fue transfundido (años 1995-1997), siendo la media 4,1 $\pm 3,9$ trGRD. Los factores que con mayor frecuencia se asociaron a transfusión fueron edad gestacional bajo $32 \mathrm{sem}$, peso de nacimiento menor o igual a $1.500 \mathrm{~g}$, membrana hialina y bronconeumonía. La exposición a donantes mostró una media de $4,1 \pm$ 3,9 donantes diferentes ${ }^{4}$.

En 1998, se elaboró y puso en marcha la guía "Recomendaciones para la Práctica Transfusional Neonatal Hospital Clínico Regional Valdivia”. En abril de ese mismo año, se inició el Programa ministerial de Surfactante pulmonar. Entre los años 2000 y 2002, estando en vigencia las recomendaciones anteriormente mencionadas y actualizada su difusión, el 77,1\% de los RNMBPN evaluados fue receptor de trGRD. El subgrupo de peso inferior a $1.000 \mathrm{~g}$ aportó con $97,1 \%$ de trGRD. La media de transfusiones y de exposición a donantes diferentes para el grupo fue 2,7 $\pm 3,2$ y los factores asociados a requerimiento transfusional fueron edad gestacional menor a 30 sem, peso menor a $1.000 \mathrm{~g}$, displasia broncopulmonar y sepsis 5 .

Hasta el momento, la acción en salud que mayor impacto ha tenido sobre el descenso del número de transfusiones, tanto en niños como adultos, es la existencia de guías para la práctica transfusional, su difusión y evaluación periódicas ${ }^{6}$. El objetivo de este trabajo es describir las características de los RNMBPN receptores de trGRD. 


\section{Pacientes y Método}

\section{Diseño del estudio}

Estudio transversal descriptivo, que incluyó las fichas clínicas de todos los RNMBPN mayores de $72 \mathrm{~h}$ de vida, egresados de Neonatología, Hospital Base Valdivia (HBV), entre el 01.01.05 y el 31.12.06. Fueron incluidos en el estudio todos aquellos neonatos de peso de nacimiento menor a $1.500 \mathrm{~g}$ y/o edad gestacional menor a $32 \mathrm{sem}$. Se excluyó a los fallecidos antes de las $72 \mathrm{~h}$ de vida y a los pacientes trasladados a otros centros.

\section{Características evaluadas}

Se evaluaron las siguientes características epidemiológicas: peso de nacimiento, edad gestacional, uso de surfactante pulmonar, diagnóstico de membrana hialina, ventilación mecánica, días de ventilación mecánica, displasia broncopulmonar, sepsis y transfusiones de GRD recibidas.

\section{Manejo transfusional de la anemia en RNMBPN}

Se utilizó la Guía para la Práctica Transfusional de GRD en Neonatos, Hospital Base Valdivia, 1998, versión 2005. En ella destaca:

Indicaciones en $R N$ en el $1^{\text {er }}$ día de vida

- Paciente con Hematocrito (Hcto) $<40 \%$ o Hemoglobina $(\mathrm{Hgb})<13 \mathrm{~g} / \mathrm{dl}$.

- Paciente con Hcto $<45 \%$ o Hgb $<14$ g/dl asociada a hemólisis.

- Requerimiento terapéutico de oxígeno.

- Paciente con Hcto $<30 \%$ o $\mathrm{Hgb}<10 \mathrm{~g} / \mathrm{dl}$, excepto aquel asintomático y con reticulocitos altos $(>3 \%)$.

Indicaciones en el RN entre $2^{\circ}$ y $14^{\circ}$ día de vida

- Paciente con Hcto $<35 \%$ o Hgb $<12$ g/dl sintomático.

Se considera sintomático presencia de:

Requerimiento de oxígeno con $\mathrm{FIO}_{2}>35 \%$. Ventilación Mecánica.

FC $>180 x^{`}$ y/o FR $>80$.

- Apneas:

- 2 episodios de apnea en $12 \mathrm{~h}$ en pacientes no tratados con Metilxantinas.

2 episodios de apnea en 24 h que requieran uso de Ambú en pacientes con niveles plasmáticos normales de Metilxantinas.

- Incremento ponderal $<10 \mathrm{~g} /$ día al menos durante 4 días, con un ingreso calórico diario $\geq 100 \mathrm{cal} / \mathrm{kg} /$ día.

- En período preoperatorio por requerimiento quirúrgico.

\section{Indicaciones después de las 2 semanas de vida}

Todo paciente con Hcto $<20 \%$ o $\mathrm{Hgb}<6$ $\mathrm{g} / \mathrm{dL}$, excepto el que esté asintomático y con reticulocitos altos $>3 \%$.

Se dispuso de GRD leucorreducidos y el volumen transfundido fue de $15 \mathrm{ml} / \mathrm{kg}$.

\section{Análisis estadístico}

Se calcularon medidas de resumen de los datos y de asociación (OR); la significación estadística se determinó mediante la dócima t de Student, con un nivel de significación alfa de $5 \%$. Los datos fueron analizados mediante el programa Epi Info versión 3.5.

Este trabajo fue aprobado por el Comité de Ética de la Facultad de Medicina, Universidad Austral de Chile.

\section{Resultados}

Entre el 1 de enero de 2005 y el 31 de diciembre de 2006, egresaron 111 RNMBPN. Reunieron los criterios de selección 93 neonatos y fueron excluidos 18 pacientes: 5 por traslado a otros centros para ventilación mecánica por falta de cupos en HBV y 13 por fallecimiento antes de las $72 \mathrm{~h}$ de vida, por patología asociada a la prematurez. En total, fueron transfundidos 62 de 93 neonatos $(66,7 \%)$. Las características de los pacientes transfundidos se muestran en la tabla 1.

Se registraron 163 episodios transfusionales para 62 RNMBPN, siendo la media de 2,1 $\pm 0,90$ trGRD. En el grupo de edad gestacional menor a $30 \operatorname{sem}(n=32)$, se observó una media de 2,5 $\pm 0,76$ trGRD versus $1,7 \pm 0,89$ en el grupo de 30 y más sem $(\mathrm{n}=30)(\mathrm{p}=0,001)$. Se observó disminución del número de transfusiones al aumentar el peso de nacimiento $(p=0,0002)($ tabla 2$)$. 
Tabla 1. Características de 62 RNMBPN transfundidos, HBV (2005-2006)

\begin{tabular}{|lcr|}
\hline Característica & $\mathbf{n}$ & \% \\
\hline Edad gestacional $\leq 30 \mathrm{~s}$ & 32 & 51,61 \\
Peso g $\quad \leq 499$ & 1 & 1,61 \\
$500-750$ & 6 & 9,67 \\
$751-1.000$ & 10 & 16,12 \\
$1.001-1.250$ & 21 & 33,87 \\
$1.251-1.500$ & 19 & 30,64 \\
$\quad \geq 1.500$ & 5 & 8,06 \\
Enfermedad membrana hialina & 45 & 72,58 \\
Uso de surfactante pulmonar & 41 & 66,12 \\
Sepsis & 27 & 43,54 \\
Ventilación mecánica & 49 & 70,03 \\
\hline
\end{tabular}

Los pacientes con diagnóstico de Membrana Hialina $(\mathrm{n}=45)$ presentaron una media de transfusiones de 2,35 $\pm 0,83$; los neonatos con diagnóstico de Distress Respiratorio no membrana hialina $(n=15)$ mostraron una media de $1,60 \pm 0,91 \operatorname{trGRD}(\mathrm{p}=0,0093)$. Se confirmó

Tabla 3. Factores asociados a trGRD en RNMBPN (2005-2006)

\begin{tabular}{|lccc|}
\hline Variable & OR & IC 95\% & Valor $\mathbf{p}$ \\
Displasia broncopulmonar & 8,05 & $1,75-36,93$ & 0,002 \\
\hline Edad gestacional < 30 sem & 5,5 & $1,86-16,19$ & 0,001 \\
Sepsis & 3,75 & $1,27-11,00$ & 0,012 \\
Membrana hialina & 3,53 & $1,42-8,80$ & 0,005 \\
Uso de surfactante & 3,45 & $1,39-8,57$ & 0,006 \\
Ventilación mecánica & 2,56 & $0,98-6,64$ & 0,048 \\
Peso al nacer < 1.000 g & 1,73 & $0,56-5,30$ & 0,32 \\
Pequeño para edad gestac. & 0,65 & $0,35-2,08$ & 0,73 \\
\hline
\end{tabular}

Tabla 2. RNMBPN transfundidos, según peso y media de transfusiones, HBV (2005-2006)

\begin{tabular}{|cccc|}
\hline Peso, g & $\begin{array}{c}\text { n de } \\
\text { RNMBPN }\end{array}$ & $\begin{array}{c}\text { Media de } \\
\text { trGRD }\end{array}$ & $\begin{array}{c}\text { Desviación } \\
\text { standard }\end{array}$ \\
\hline$<499$ & 1 & 3,00 & 0,00 \\
\hline $500-750$ & 6 & 3,00 & 0,00 \\
\hline $751-1.000$ & 10 & 2,80 & 0,63 \\
\hline $1.001-1.250$ & 21 & 1,76 & 0,88 \\
\hline $1.251-1.500$ & 19 & 2,15 & 0,83 \\
\hline$>1.500$ & 5 & 1,20 & 0,44 \\
\hline Total & 62 & & \\
\hline
\end{tabular}

sepsis en 27 neonatos, con una media de transfusiones de 2,48 $\pm 0,84$; en 35 neonatos que no cursaron sepsis, se observó una media de 1,88 $\pm 0,86 \operatorname{trGRD}(\mathrm{p}=0,0088)$.

El 33,87\% de los neonatos no fue usuario de surfactante pulmonar, y en este grupo la media de transfusiones observada fue 1,57 \pm 0,81 versus $2,43 \pm 0,80$ trGRD en el grupo que recibió surfactante pulmonar $(\mathrm{p}=0,0002)$. El $79,03 \%$ de este grupo de RNMBPN requirió ventilación mecánica con 2,38 $\pm 0,81$ trGRD, versus 1,23 $\pm 0,59$ trGRD en el grupo de 13 $(20,96 \%)$ RNMBPN que no la requirió ( $p$ $=0,0000)$.

De este modo los factores asociados a trGRD incluyeron displasia broncopulmonar, edad gestacional $<30$ sem, presencia de sepsis, membrana hialina, uso de surfactante y requerimiento de ventilación mecánica (tablas 3 y 4). No se encontró asociación entre peso según edad gestacional y número de transfusiones.

Tabla 4. Factores protectores para trGRD en RNMBPN (2005-2006)

\begin{tabular}{|lrrcrc|}
\hline Variable & $\mathbf{n}$ & $\mathbf{\%}$ & Media trGRD & DS & p \\
EG $>$ 30 sem & 30 & 48,38 & 1,76 & 0,89 & 0,0010 \\
Peso nac $>1.500 \mathrm{~g}$ & 5 & 8,06 & 1,20 & 0,44 & 0,0002 \\
Sin falla respiratoria & 15 & 24,19 & 1,60 & 0,91 & 0,0093 \\
Ausencia de sepsis & 35 & 56,45 & 1,88 & 0,86 & 0,0088 \\
Sin requerimiento de surfactante & 21 & 33,87 & 1,57 & 0,81 & 0,0002 \\
Sin requerimiento ventilatorio & 13 & 20,96 & 1,23 & 0,35 & 0,0000 \\
\hline
\end{tabular}




\section{Discusión}

En esta serie de RNMBPN se transfundió el $66,7 \%$ de los pacientes (62 de 93), recibiendo $2,1 \pm 0,9$ trGRD; al carecer de sistemas de alicuotación de unidades de GRD en el momento del estudio, la exposición a donantes diferentes fue de 2,1 $\pm 0,9$. Los resultados obtenidos muestran un descenso progresivo en el porcentaje de RNMBPN transfundidos en las series evaluadas: $89,4 \%$ en $1995,77,1 \%$ el año 2000 y $66,7 \%$ el año 2005 . Las medias de transfusiones observadas en estos grupos fueron 4,1 trGRD $\pm 3,9$ en $1995 ; 2,7 \operatorname{trGRD} \pm 3,2$ el año 2000 y 2,1 trGRD $\pm 0,90$ el año $2005^{4,5}$, resultados que puede atribuirse a la difusión y conocimiento de las recomendaciones de transfusión.

En concordancia con la literatura, los RNMBPN con peso de $1.000 \mathrm{~g}$ o menos y aquellos con edad gestacional menor a $30 \mathrm{sem}$, son los que aportan el mayor número de casos al grupo de transfundidos, probablemente en relación a la anemia del prematuro agravada por las extracciones de sangre para estudio durante su hospitalización, por lo que resultan de interés tanto la indicación cuidadosa de estudio de laboratorio, como el uso de micrométodos ${ }^{1,6,7}$.

La media de trGRD en RNMBPN, que en estudios previos mostró un descenso importante el año 2000 respecto del año 1995, tiende a mantenerse entre 2,5 y 2,1 , probablemente en relación a una mejor adherencia y difusión de las recomendaciones para transfundir, especialmente a los nuevos integrantes del equipo de salud, tanto como a la auditoría de la práctica transfusional realizada por el Comité de Medicina Transfusional del HBV, lo que sugiere que en este grupo de pacientes es necesario, sin desmedro de lo anterior, agregar estrategias que resulten en disminución de la exposición a transfusiones, como por ejemplo retardar el pinzamiento del cordón umbilical, ampliar el uso de micrométodos de laboratorio, monitorear en forma no invasiva ${ }^{2,3}$ y en casos seleccionados usar eritropoyetina, ya que su uso, asociado a sulfato ferroso y ácido fólico, disminuye el requerimiento transfusional ${ }^{8,9}$. En el futuro será de interés la evaluación de la implementación de trGRD autólogos de sangre de cordón almacenada en bancos, especialmente en neonatos con factores de riesgo asociados a requerimiento transfusional ${ }^{10,11}$.

Ante el requerimiento de trGRD homólogos, la alicuotación y reserva de una unidad de concentrado eritrocitario para un receptor particular en riesgo de recibir una segunda o tercera transfusión, permite disminuir la exposición a diferentes donantes de sangre; la disponibilidad de hemocomponentes leucorreducidos, filtrados o irradiados, disminuiría el riesgo de transmisión de infecciones asociadas a transfusiones y otras reacciones adversas a las transfusiones, entre ellas enfermedad injerto versus huésped, en particular en susceptibles como los prematuros.

Los factores asociados al riesgo de trGRD en este grupo de neonatos y su contrapartida constituyen factores protectores para el requerimiento transfusional, por lo que sugerimos especial atención a los programas de la autoridad sanitaria para la prevención y pesquisa del parto prematuro y de la falla respiratoria por membrana hialina.

En conclusión, el perfil del RNMBPN susceptible de ser receptor de trGRD en esta serie de pacientes es aquel de EG $<30$ sem, peso inferior a $1.250 \mathrm{~g}$, con falla respiratoria, ventilación mecánica y sepsis. Se aprecia un descenso progresivo en los porcentajes de RNMBPN transfundidos comparados con datos históricos, en la media de trGRD y la de exposición a donantes diferentes respecto de otras series de de RNMBPN evaluados previamente, que podrían ser atribuidos a la existencia de guías para la práctica transfusional neonatal, a su difusión, a la adherencia por un equipo de salud altamente sensibilizado, tanto como a la auditoría periódica de la práctica transfusional.

\section{Referencias}

1.- Strauss R: Transfusion therapy in neonates. Am J Dis Chil 1991; 145: 904-1.

2.- Shannon K, Keith J, Mentzer R, Ehrenkranz R, Brown, et al: Recombinan Human Erythropietin Stimulates Erythropoiesis and Reduces Erythrocyte Transfusions in Very Low Birth Weight Preterm Infants. Pediatrics 1995; 95: 1-8. 
3.- Von Kohorn I, Ehrenkranz R: Anemia in the Preterm Infant: Erythropoietin versus Erythrocyte Transfusion: It's not that Simple. Clin Perinatol 2009; 36: 111-23.

4.- Lagos M, Hering V, Marín F, Zolezzi P: Transfusión de glóbulos rojos en recién nacidos de muy bajo peso (RNMBP) Hospital Clínico Regional de Valdivia. Rev Chil Pediatr 2000; 71: 413-8.

5.- Folatre I, Scheuch K, Hesse H, et al: Evaluación de Guías para la Práctica Transfusional Neonatal Hospital Clínico Regional Valdivia (2000-2002). Rev Chil Pediatr 2004; 75: 565-641.

6.- Corwin H, Carson J: When is More Really Less? N Engl J Med 2007; 356; 16.

7.- Valieva O, Stransjord T, Mayock D, Juul S: Effects of Transfusions in Extremely Low Birth Weight Infants: A Retrospective Study. The J Ped 2009; 155: 331-7.

8.- Whyte R, Kirpalani H, Asztalos E, et al: PINTOS Study
Group. Neurodevelopmental Outcome of Extremely Low Birth Weight Infants randomly assigned to restrictive or liberal hemoglobin thresholds for blood transfusion. Pediatrics 2009; 123: 207-13.

9.- Guzmán J, De La Torre M, Tofe I, et al: Factores de Riesgo implicados en la necesidad de transfusión sanguínea en recién nacidos de muy bajo peso de nacimiento tratados con Eritropoyetina. An Pediatr (Barc) 2010; 73: 340-6.

10.- Narendra A, McHugh S, Aitchison T, Wardrop C, Holland B: Infant's Blood Volume in a Controlled Trial of Placental Transfusion at Preterm Delivery. Pediatrics 2006; 117; 93-8.

11.- Donald G, Strauss MD, John Widness MD: Is There a Rol for Autologous Placental RBC Transfusion in the Anemia of Prematurity? Transfusion Med Rev 2010; 24: $125-9$. 